\title{
Virological COVID-19 surveillance in Bavaria, Germany suggests no SARS-CoV-2 spread prior to the first German case in January 2020
}

\author{
Ute Eberle $^{1} \cdot$ Susanne Heinzinger ${ }^{2} \cdot$ Regina Konrad $^{1} \cdot$ Clara Wimmer $^{1} \cdot$ Bernhard Liebl $^{3,4} \cdot$ Katharina Katz $^{5}$. \\ Nikolaus Ackermann ${ }^{1}$. Andreas Sing ${ }^{2,3,4}$ - Bavarian SARS-CoV-Public Health Laboratory and Epidemiology Team
}

Received: 23 February 2021 / Accepted: 27 March 2021 / Published online: 23 April 2021

(c) The Author(s) 2021

\begin{abstract}
The Bavarian Influenza Sentinel (BIS) monitors the annual influenza season by combining virological and epidemiological data. The 2019/2020 influenza season overlapped with the beginning COVID-19 pandemic thus allowing to investigate whether there was an unnoticed spread of SARS-CoV-2 among outpatients with acute respiratory infections in the community prior to the first COVID-19 cluster in Bavaria. Therefore, we retrospectively analysed oropharyngeal swabs obtained in BIS between calendar week (CW) 39 in 2019 and CW 14 in 2020 for the presence of SARS-CoV-2 RNA by RT-PCR. 610 of all 1376 BIS swabs-contained sufficient material to test for SARS-CoV-2, among them 260 oropharyngeal swabs which were collected prior to the first notified German COVID-19 case in CW 04/2020. In none of these swabs SARS-CoV-2 RNA was detected suggesting no SARS-CoV-2 spread prior to late January 2020 in Bavaria.
\end{abstract}

Keywords Influenza $\cdot$ COVID-19 $\cdot$ SARS-CoV-2 $\cdot$ RT-PCR $\cdot$ Surveillance

\section{Introduction}

The COVID-19 outbreak in Wuhan/China at the end of December 2019 led to a rapid spread of SARS-CoV-2 throughout the world [1-3]. In calendar week (CW) 5, 2020 (Jan 27, 2020), the Bavarian Health and Food Safety Authority (LGL), was informed about the first human SARS-CoV-2 infection in Germany [4-7] leading to a cluster of several

Ute Eberle and Susanne Heinzinger contributed equally.

Nikolaus Ackermann and Andreas Sing contributed equally.

Andreas Sing

andreas.sing@1gl.bayern.de

1 Unit of Virology, Bavarian Health and Food Safety Authority, Oberschleißheim, Germany

2 Unit of Public Health Microbiology, Bavarian Health and Food Safety Authority, Bayerisches Landesamt Für Gesundheit Und Lebensmittelsicherheit (LGL), Veterinärstraße 2, 85764 Oberschleißheim, Germany

3 State Institute of Health, Bavarian Health and Food Safety Authority, Oberschleißheim, Germany

4 Ludwig Maximilians-Universität, Munich, Germany

5 Unit of Infectious Diseases Epidemiology, Bavarian Health and Food Safety Authority, Oberschleißheim, Germany infected persons which could be contained by an immediate public health response [4, 5]. The first COVID-19 cases within the WHO European Region were reported in France on January 24, 2020 with the onset of symptoms in the first patient on January 17 [6]. On March 11, 2020 WHO declared COVID-19 a pandemic [1, 2, 4].

Due to the lack of sufficient RT-PCR methods for SARS$\mathrm{CoV}-2$ detection in the early period of the pandemic, mainly symptomatic patients were tested and subsequently identified. The increasing availability of RT-PCR tests allowed to address issues regarding the time of origin and early spread of SARS-CoV-2 which were triggered by both public and scientific interests: (i) mainly at the beginning of the pandemic, misinformation claiming an already long-standing spread of SARS-CoV-2 months or even years before the end of 2019 was disseminated, partly with the aim to raise doubt about the necessity of public health measures towards a supposedly pre-existing pathogen [7]; (ii) phylogenetic analysis of genomic data estimated the start of the COVID-19 pandemic in the period of October 6 to December 11, 2019 [8], dating back several weeks before the first clinical human cases were detected in Wuhan/China; (iii) retrospective testing of respiratory material from intensive care influenza-like illness (ILI) patients in a hospital from the Paris area identified a COVID-19 patient becoming symptomatic on December 27, 
2019 nearly three weeks before the first reported COVID-19 case in France [9]; (iv) environmental monitoring studies finding SARS-CoV-2 RNA in wastewater dating back to December 18, 2019 suggested SARS-CoV-2 circulation in Italy two months earlier than the first reported autochthonous Italian case [10].

During the influenza season 2019/2020, the Bavarian Influenza Sentinel (BIS) established in 2009 [11] was carried out to know the proportion of ILI among patients with acute respiratory infections (ARI) caused by influenza viruses and respiratory syncytial virus (RSV).

Prompted by the issues mentioned above we retrospectively analysed BIS oropharyngeal swabs from season 2019/2020 for the presence of SARS-CoV-2 RNA to test the hypothesis of cryptic SARS-CoV-2 spread among ARI outpatients in the community.

\section{Methods}

\section{Specimen collection}

BIS consists of approximately 75 general practitioners. On a weekly basis each practitioner took specimens (naso- or oropharyngeal swabs) from two randomly chosen ARI patients (one swab per patient) and sent them to the LGL for virological diagnosis [11].

\section{Laboratory diagnostics}

Virus transport media from swabs obtained for lab-based influenza surveillance within BIS were immediately stored after initial influenza testing at $-20^{\circ} \mathrm{C}$. For SARS-CoV-2 diagnosis RNA was extracted from stored samples as previously reported [12]. The following methods were used: RNAdvance Viral Kit on a Biomeki7 (Beckman Coulter, Indianapolis, USA), MagBind Viral RNA XPRESS Kit (Omega Bio-Tek) on a Hamilton Microlab Starlet (Hamilton, Bonaduz, Switzerland) followed by the use of AmpliCube Coronavirus SARS-CoV-2 PCR Kit (Mikrogen, Neuried, Germany) on the Bio-Rad CFX96 Touch Real-Time PCR Detection System (Bio-Rad, Feldkirchen, Germany). The assay detects the E-Gene of B-lineage betacoronavirus in FAM and the Orf1a-Gene specific for SARSCoV-2 in HEX [12]. The threshold was manually set within the exponential phase of the detection curve.

\section{Data analysis, graphics}

Data were evaluated and graphics were created using Spyder 4.1.4. (Python 3.8).

\section{Results}

From CW 39/2019 to CW 14/2020 1,376 specimens were analysed for influenza viruses. 610 of these samples for which sufficient material was available, were tested for SARS-CoV-2, among them 260 oropharyngeal swabs which were collected between CW 39/2019 and CW 04/2020 prior to the first notified German COVID-19 case. No SARS-CoV2-positive results were obtained throughout the complete BIS 2019/2020 season (Fig. 1).

\section{Discussion}

Some community-based sentinels or hospital-based patient surveys conducted outside Europe, e.g. Wuhan/China [13] and the US [14-16], retrospectively analysed respiratory samples mainly from ARI or ILI patients. The Wuhan [13] and Seattle/WA study sites [14] failed to identify SARSCoV-2-RNA in 520 and approximately 400 samples, respectively, obtained prior to the first national confirmed COVID-19 case. Moreover, 11,400 samples from six US states prospectively collected within the month after the first verified US COVID-19 case did not render a SARS-CoV2-positive result [15]. Similarly, a retrospective screening study on 1,700 nasopharyngeal samples from ILI patients in California covering the last two months of 2019 failed to reveal any SARS-CoV-2 positive patient [16].

European studies analysing respiratory samples from the period before a first national COVID-19 case was notified are even fewer. A French study on 14 intensive care ILI patients identified a COVID-19 patient retrospectively having become symptomatically at the end of December 2019, about three weeks prior to the first officially confirmed

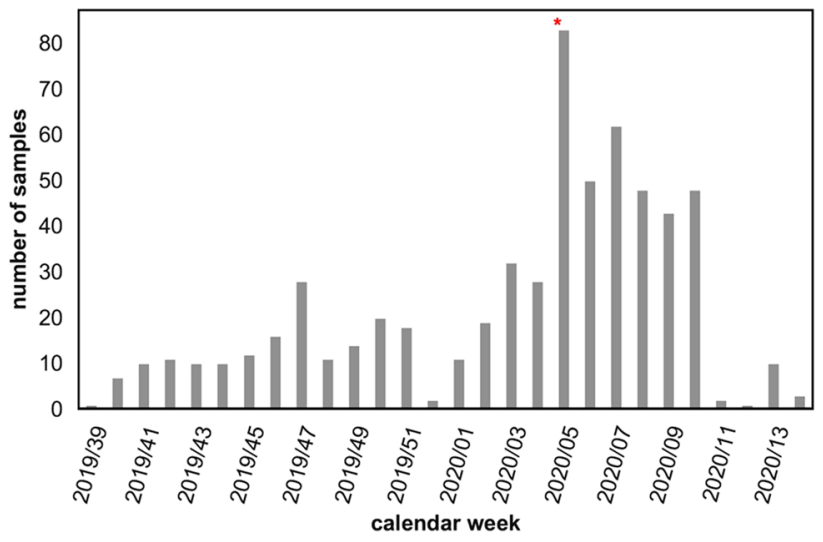

Fig. 1 Number of samples analysed for SARS-CoV-2 by calendar week in the BIS 2019/2020 season. Grey bar: negative swabs, red star: first notified German COVID-19 case on 27.01.2020 
French case [9]. To the best of our knowledge, our study has retrospectively analysed the largest number of ARI patients from a community-based sentinel for SARS-CoV-2 infection by RT-PCR so far. In contrast to the French study [9], we could not identify a SARS-CoV-2-positive sample among 260 oropharyngeal swabs obtained between CW $39 / 2019$ and $04 / 2020$ prior to the first German case identified on January 27, 2020. Similarly, a retrospective analysis of 195 respiratory samples from the German CAPNETZ cohort of community-acquired pneumonia obtained during the 2019/2020 influenza season between December 2, 2019 (CW 48/2019) and April 28, 2020 (CW 17/2020) did not detect any SARS-CoV-2-infection before March 24, 2020 [17].

Limitations of our study are mainly due to its necessarily retrospective design relying on stored material. In addition, the BIS although comprising about 75 private medical physicians from all parts of Bavaria is not completely representative for the Bavarian population.

In conclusion, our study supports the hypothesis that there was no notable circulation of SARS-CoV-2 in the Bavarian population before the first German COVID-19 cluster at the end of January 2020.

Acknowledgements We want to thank for excellent technical assistance in diagnostic and validation lab work: Evelyn Bauermeister, Magdalena Bichler, Sigrun Boehmer, Juliane Breitenberger, Turgut-Cengiz Dedeoglu, Karin Drexl Jessica Feldmann, Jasmin Fräßdorf, Christine Hartberger, Angela Huber, Sabine Lohrer, Renate Kloos, Nigar Krüss, Bernhard Müller, Linda Ploß, Hermine Roth, Monika Schmittner, Larissa Schulz, Renate Seebeck, Annemarie Seiler, Claudia Stapel, Christiane Strauß, Lisa Thoms, Ayfer Yardim, Peggy Zill and all others who contributed to sample and lab logistics.

Authors' contributions UE created the study concept, conducted the analysis, and drafted the manuscript. SH created the study concept, conducted the analysis. RK, CW performed RT-PCR testing and analysis. All authors participated in the interpretation of data as well as critical revisions of the manuscript. NA, KK, and AS supervised the study.

Funding Open Access funding enabled and organized by Projekt DEAL. This work was supported by Bavarian Health and Food Safety Authority and the European Centre for Disease Prevention and Control.

Availability of data and materials Data available within the article or its supplementary materials.

Code availability Not applicable.

\section{Declarations}

Ethics approval Ethics approval was obtained (Ethik-Kommission Nr. 15053).

Consent to participate The patients were informed in a "Patienteninformation" about the procedure of the BIS and confirmed their participation with a signature.
Consent for publication The patients were informed in a "Patienteninformation" about the possibility to publicise aggregated BIS data and confirmed this with their signature.

Conflicts of interest The authors report no potential conflicts of interest.

Open Access This article is licensed under a Creative Commons Attribution 4.0 International License, which permits use, sharing, adaptation, distribution and reproduction in any medium or format, as long as you give appropriate credit to the original author(s) and the source, provide a link to the Creative Commons licence, and indicate if changes were made. The images or other third party material in this article are included in the article's Creative Commons licence, unless indicated otherwise in a credit line to the material. If material is not included in the article's Creative Commons licence and your intended use is not permitted by statutory regulation or exceeds the permitted use, you will need to obtain permission directly from the copyright holder. To view a copy of this licence, visit http://creativecommons.org/licenses/by/4.0/.

\section{References}

1. Yesudhas D, Srivastava A, Gromiha MM. COVID-19 outbreak: history, mechanism, transmission, structural studies and therapeutics. Infection. 2020. https://doi.org/10.1007/s15010-020-01516-2.

2. Salzberger B, Buder F, Lampl B, et al. Epidemiology of SARS-CoV-2. Infection. 2020. https://doi.org/10.1007/ s15010-020-01531-3.

3. Hoffmann C, Wolf E. Older age groups and country-specific case fatality rates of COVID-19 in Europe. USA and Canada Infection. 2021;49:111-6.

4. Böhmer MM, Buchholz U, Corman VM, Hoch M, Katz K, Marosevic DV, et al. Investigation of a COVID-19 outbreak in Germany resulting from a single travel-associated primary case: a case series. Lancet Infect Dis. 2020;20:920-8.

5. Konrad R, Eberle U, Dangel A, Treis B, Berger A, Bengs K, et al. Rapid establishment of laboratory diagnostics for the novel coronavirus SARS-CoV-2 in Bavaria, Germany, February 2020. Euro Surveill. 2020;25(9):2000173.

6. Spiteri G, Fielding J, Diercke M, Campese C, Enouf V, Gaymard A, et al. First cases of coronavirus disease 2019 (COVID-19) in the WHO European Region, 24 January to 21 February 2020. Euro Surveill. 2020;25:2000178.

7. Lu J. Themes and Evolution of Misinformation During the Early Phases of the COVID-19 Outbreak in China-An Application of the Crisis and Emergency Risk Communication Model. Front Commun. 2020. https://doi.org/10.3389/fcomm.2020.00057.

8. van Dorp L, Acman M, Richard D, Shaw LP, Ford CE, Ormond $\mathrm{L}$, et al. Emergence of genomic diversity and recurrent mutations in SARS-CoV-2. Infect Genet Evol. 2020;83:104351.

9. Deslandes A, Berti V, Tandjaoui-Lambotte Y, Alloui C, Carbonnelle E, Zahar JR, et al. SARS-CoV-2 was already spreading in France in late December 2019. Int J Antimicrob Agents. 2020;55:106006.

10. La Rosa G, Mancini P, Bonanno Ferraro G, Veneri C, Iaconelli M, Bonadonna L, et al. SARS-CoV-2 has been circulating in northern Italy since December 2019: evidence from environmental monitoring. Sci Total Environ. 2021;750:141711.

11. Campe H, Heinzinger S, Hartberger C, Sing A. Clinical symptoms cannot predict influenza infection during the 2013 influenza season in Bavaria. Germany Epidemiol Infect. 2016;144:1045-51.

12. Eberle U, Wimmer C, Huber I, et al. Comparison of nine different commercially available molecular assays for detection of 
SARS-CoV-2 RNA. Eur J Clin Microbiol Infect Dis. 2021. https:// doi.org/10.1007/s10096-021-04159-9.

13. Kong WH, Li Y, Peng MW, Kong DG, Yang XB, Wang L, et al. SARS-CoV-2 detection in patients with influenza-like illness. Nat Microbiol. 2020;5:675-8.

14. Chu HY, Englund JA, Starita LM, Famulare M, Brandstetter E, Nickerson DA, et al. Early detection of Covid-19 through a citywide pandemic surveillance platform. N Engl J Med. 2020;383:185-7.

15. Jorden MA, Rudman SL, Villarino E, Hoferka S, Patel MT, Bemis $\mathrm{K}$, et al. Evidence for limited early spread of COVID-19 within the United States, January-February 2020. MMWR Morb Mortal Wkly Rep. 2020;69:680-4.

16. Hogan CA, Garamani N, Sahoo MK, Huang C, Zehnder J, Pinsky BA. Retrospective screening for SARS-CoV-2 RNA in California, USA, Late 2019. Emerg Infect Dis. 2020;26:2487-8.

17. Panning M, Wiener J, Rothe K, et al. No SARS-CoV-2 detection in the German CAPNETZ cohort of community acquired pneumonia before COVID-19 peak in March 2020. Infection. 2020;48:971-4. 